\title{
Secondary threshold amplitudes for sinuous streak breakdown
}

\author{
Carlo Cossu, ${ }^{1, a)}$ Luca Brandt, ${ }^{2}$ Shervin Bagheri, ${ }^{2}$ and Dan S. Henningson ${ }^{2}$ \\ ${ }^{1}$ IMFT - CNRS, Allée du Professeur Camille Soula, 31400 Toulouse, France \\ ${ }^{2}$ KTH Mechanics \& Linné Flow Center, S-10044 Stockholm, Sweden
}

(Received 4 March 2011; accepted 20 June 2011; published online 26 July 2011)

\begin{abstract}
The nonlinear stability of laminar sinuously bent streaks is studied for the plane Couette flow at $R e=500$ in a nearly minimal box and for the Blasius boundary layer at $R e_{\delta^{*}}=700$. The initial perturbations are nonlinearly saturated streamwise streaks of amplitude $A_{U}$ perturbed with sinuous perturbations of amplitude $A_{W}$. The local boundary of the basin of attraction of the linearly stable laminar flow is computed by bisection and projected in the $A_{U}-A_{W}$ plane providing a well defined critical curve. Different streak transition scenarios are seen to correspond to different regions of the critical curve. The modal instability of the streaks is responsible for transition for $A_{U}=25 \%-27 \%$ for the considered flows, where sinuous perturbations of amplitude below $A_{W} \approx 1 \%-2 \%$ are sufficient to counteract the streak viscous dissipation and induce breakdown. The critical amplitude of the sinuous perturbations increases when the streamwise streak amplitude is decreased. With secondary perturbations amplitude $A_{W} \approx 4 \%$, breakdown is induced on stable streamwise streaks with $A_{U} \approx 13 \%$, following the secondary transient growth scenario first examined by Schoppa and Hussain [J. Fluid Mech. 453, 57 (2002)]. A cross-over, where the critical amplitude of the sinuous perturbation becomes larger than the amplitude of streamwise streaks, is observed for streaks of small amplitude $A_{U}<5 \%-6 \%$. In this case, the transition is induced by an initial transient amplification of streamwise vortices, forced by the decaying sinuous mode. This is followed by the growth of the streaks and final breakdown. The shape of the critical $A_{U}-A_{W}$ curve is very similar for Couette and boundary layer flows and seems to be relatively insensitive to the nature of the edge states on the basin boundary. The shape of this critical curve indicates that the stability of streamwise streaks should always be assessed in terms of both the streak amplitude and the amplitude of spanwise velocity perturbations. (C) 2011 American Institute of Physics.
\end{abstract}

[doi:10.1063/1.3614480]

\section{INTRODUCTION}

Streamwise streaks are narrow regions of excess or defect streamwise velocity that are elongated in the streamwise direction. Their existence was first remarked in boundary layers forced by free-stream-turbulence ${ }^{1-3}$ and it was then realised that they play an essential role in the buffer layer of turbulent shear flows. ${ }^{4}$ Further investigations have revealed the presence of these structures at all scales in virtually all shear flows in the transitional and turbulent regimes. Streamwise streaks are very efficiently generated from streamwise vortices via the lift-up effect. ${ }^{5,6}$ The ratio of the energy of the streaks compared to the energy of the vortices that have generated them can reach values of the order of the square of the Reynolds number ${ }^{7}$ even with a stable base flow. These very large energy amplifications are related to the strongly non-normal nature of the linearized NavierStokes operator for shear flows, ${ }^{8,9}$ and the associated transient energy growths and sustained harmonic responses have been computed for all the canonical shear flows.

Streamwise uniform streaks of large enough amplitude are known to become unstable via an inviscid inflectionaltype mechanism. ${ }^{10-13}$ In laminar flows, the unstable modes are streamwise non-uniform with a streamwise wavelength of the order of the spanwise wavelength of the streaks. Usually,

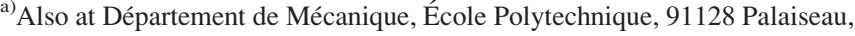
France.
}

the first unstable modes are sinuous and have the same spanwise wavelength of the streaks (fundamental modes). The instability of the streaks is important because the amplification of the secondary mode leading to the streak breakdown allows to regenerate streamwise vorticity and to close the loop of self-sustained processes. It was indeed proposed that such an endogenous self-sustained process is essential in the understanding of turbulent dynamics in the near-wall region, ${ }^{14,15}$ and it has been shown that a similar scenario is likely to induce subcritical transition in laminar channel flows. ${ }^{11,16}$

The original view that a secondary modal instability of the streaks is necessary for their breakdown has been questioned by Schoppa and Hussain. ${ }^{17}$ These authors use data extracted from near-wall direct numerical simulations (DNS) of turbulent channel flows and note that only less than $20 \%$ of the near-wall streaks exceeds the critical amplitude for secondary instability. They suggest as relevant breakdown mechanism the transient growth of secondary perturbations riding on top of modally stable streaks. Optimal secondary transient growths have been computed for boundary layer and channel flows streaks. ${ }^{18,19}$ Similar considerations arise in the case of the transition induced by free-stream turbulence in the flat plate boundary layer where transition to turbulence is observed for streak amplitudes below the critical threshold for secondary instability. Also in this case, alternative mechanisms have been proposed to bypass the secondary instability of the streaks. ${ }^{20,21}$ For instance, Brandt et al..$^{20}$ perform 
numerical simulations of collision of finite-length streaks and show that this is able to initiate the breakdown below the critical amplitudes.

The recent analyses of the instability of linearly stable streaks can be rationalized by recognizing the subcritical nature of the streaks breakdown, e.g., revealed by the continuation procedure used by Waleffe $\mathrm{e}^{22,23}$ to find self-sustained nonlinear states. If streaks of large enough amplitude, above the critical one, will experience breakdown even in the presence of infinitesimal perturbations, linearly stable streaks will need perturbations of finite amplitude to undergo breakdown, and indeed, finite amplitude perturbations were considered in previous investigations of streaks breakdown. ${ }^{17,20}$

In all the previous studies on secondary streak transient growth and streak breakdown, however, a systematic quantitative analysis of the critical secondary perturbation amplitudes is missing and it is, therefore, difficult to appreciate the actual relevance of the proposed subcritical scenarios. The scope of the present study is therefore to quantify the breakdown boundary in terms of the amplitude of the streaks and of their secondary perturbations. The secondary perturbations will be assumed to be sinuous, as this is the shape of the critical unstable mode and of the perturbations observed in the breakdown. This is equivalent to investigate the shape of the boundary of the basin of attraction of the laminar solution for a two-parameter family of sinuously bent streaks.

We introduce the main processes we are interested in with the help the simple toy model of Waleffe ${ }^{10}$ in Sec. I. For this model, we determine the local boundary of the basin of attraction of the laminar solution in terms of amplitudes of the streamwise streak and of its spanwise perturbation. The relation between the local basin boundary and the lower branch saddle solutions, the edge state of this system, is also discussed on the toy model. The main ideas discussed in Sec. I are then applied to the plane Couette flow at $R e=500$ and to the Blasius boundary layer at $R e_{\delta^{*}}=700$ in Secs. II and III, respectively. Finally, the main implications of the results are discussed in Sec. IV.

\section{INTRODUCTORY ANALYSIS ON A TOY MODEL}

We first develop our arguments on the simple fourdimensional model system proposed by Waleffe $\mathrm{e}^{10,24}$

$$
\begin{gathered}
d u / d t=-\left(k_{u}^{2} / R\right) u+\sigma_{u} v-\sigma_{w} w^{2}+\sigma_{u} m v, \\
d v / d t=-\left(k_{v}^{2} / R\right) v+\sigma_{v} w^{2}, \\
d w / d t=\left[-\left(k_{w}^{2} / R\right)+\sigma_{w} u-\sigma_{m} m-\sigma_{v} v\right] w, \\
d m / d t=-\left(k_{m}^{2} / R\right) m-\sigma_{u} u v,
\end{gathered}
$$

The system is designed to mimic the nonlinear dynamics of streamwise vortices of amplitude $v$, streamwise streaks of amplitude $u$, sinuous perturbations to the streaks of amplitude $w$ and the change $m$ to the mean shear induced by these perturbations at the Reynolds number $R$. The same coefficients as those considered in Ref. 24 have been selected ([ $k_{m}$, $\left.k_{u}, k_{v}, k_{w}\right]=[1.57,2.28,2.77,2.67]$ and $\left[\sigma_{u}, \sigma_{v}, \sigma_{w}\right]=[1.29$, $0.22,0.68]$ ) with the exception of the choice $\sigma_{m}=0$, as in
Ref. 15. We define the state vector as $\mathrm{u}=[u, v, w, m]$. The "laminar solution" $\mathrm{u}=0$ is linearly stable for all $R$, but the linear non-normal coupling $\sigma_{u} v$ in the equation for the evolution of the streaks $u$ sustains optimal transient energy growths $^{25}$ proportional to $R^{2}$ at sufficiently large $R$. The optimal linear initial condition is essentially composed of streamwise vortices $\left(\mathbf{u}_{V}^{(o p t)} \approx[0,1,0,0]\right)$ and the corresponding optimal output is essentially composed of streamwise streaks $\left(\mathbf{u}_{U}^{(\text {opt })} \approx[1,0,0,0]\right)$.

Following the approach used in a number of previous studies, ${ }^{13,18,19,26,27}$ nonlinear primary streaky base flow can be generated by using the linear optimal initial perturbations with finite amplitude $A_{1} \mathbf{u}_{V}^{(o p t)}$ as initial condition for the nonlinear system (1). Typical examples of the streak transient nonlinear growth for two selected amplitudes $A_{1}$ are reported in panels (a) and (b) (solid, red line) of Fig. 1. Near the times of maximum growth the solutions are of the form $\mathrm{U}_{1} \approx[U, 0,0, M]$.

Analysis of the eigenvalues of the linearized operator show that $U_{1}$ is (locally in time) unstable when $U>U_{c}=k_{w}^{2} /\left(R \sigma_{w}\right)$ with the unstable mode $\mathrm{u}_{W}=[0,0,1,0]$. $U_{c}$, therefore, represents the critical amplitude of streaks, above which secondary instabilities can grow leading to streak breakdown and transition. As the primary base flow is actually decaying for $t=t_{\text {max }}$, values of $U$ well above $U_{c}$ are often required to trigger the streak breakdown ${ }^{11}$ with very low initial amplitudes of the secondary mode $\mathrm{u}_{W}$, especially at low or moderate $R$. When $U<U_{c}$, the primary streaks are linearly stable but recognizing that the streak instability is subcritical, one should be able to observe breakdown for suitable perturbations of sufficiently large amplitude. To investigate this issue, we therefore add a secondary perturbation $A_{2} \mathrm{u}_{W}$, a sinuous mode, to the streaky primary baseflows $\mathrm{U}_{1}$ at the time of their maximum amplitude (the small triangles in Fig. 1) and track their long-time evolution. When the secondary perturbation is too small, the solution relaxes to the laminar solution, while for sufficiently large secondary perturbation amplitudes, the solution is attracted to a finite equilibrium point, corresponding to an upper branch solution $\mathrm{u}_{U B}$ in the terminology of Waleffe. ${ }^{10,24}$ The critical secondary perturbation amplitude is found by bisection. ${ }^{11,16}$

As seen in Fig. 1, if the bisection is sufficiently refined, the solutions remain for a finite time on the boundary of the basin of attraction of the laminar solution and are attracted to the lower branch saddle solution $\mathrm{u}_{L B}$ before eventually escape to the upper branch solution or to the laminar solution. Here, $\mathrm{u}_{L B}$ represents the "edge state" of the system. ${ }^{28-30}$

The saddle point $\mathrm{u}_{L B}$ is approached in different ways, depending on the initial primary streak amplitude. For a primary base flow with primary streak amplitude well above $U_{c}$, the "classical" scenario is observed where the growth of the secondary unstable perturbations $(w)$ is sustained even during the initial decay of the primary streaks, before approaching the lower branch solution and finally escaping it (panels $a$ and $c$ in Fig. 1). When the primary streaks are stable $\left(U_{1}<U_{c}\right)$, a secondary perturbation with larger amplitude is needed to undergo transition. In this case, the lower branch is approached after an initial decrease of $w$, the 

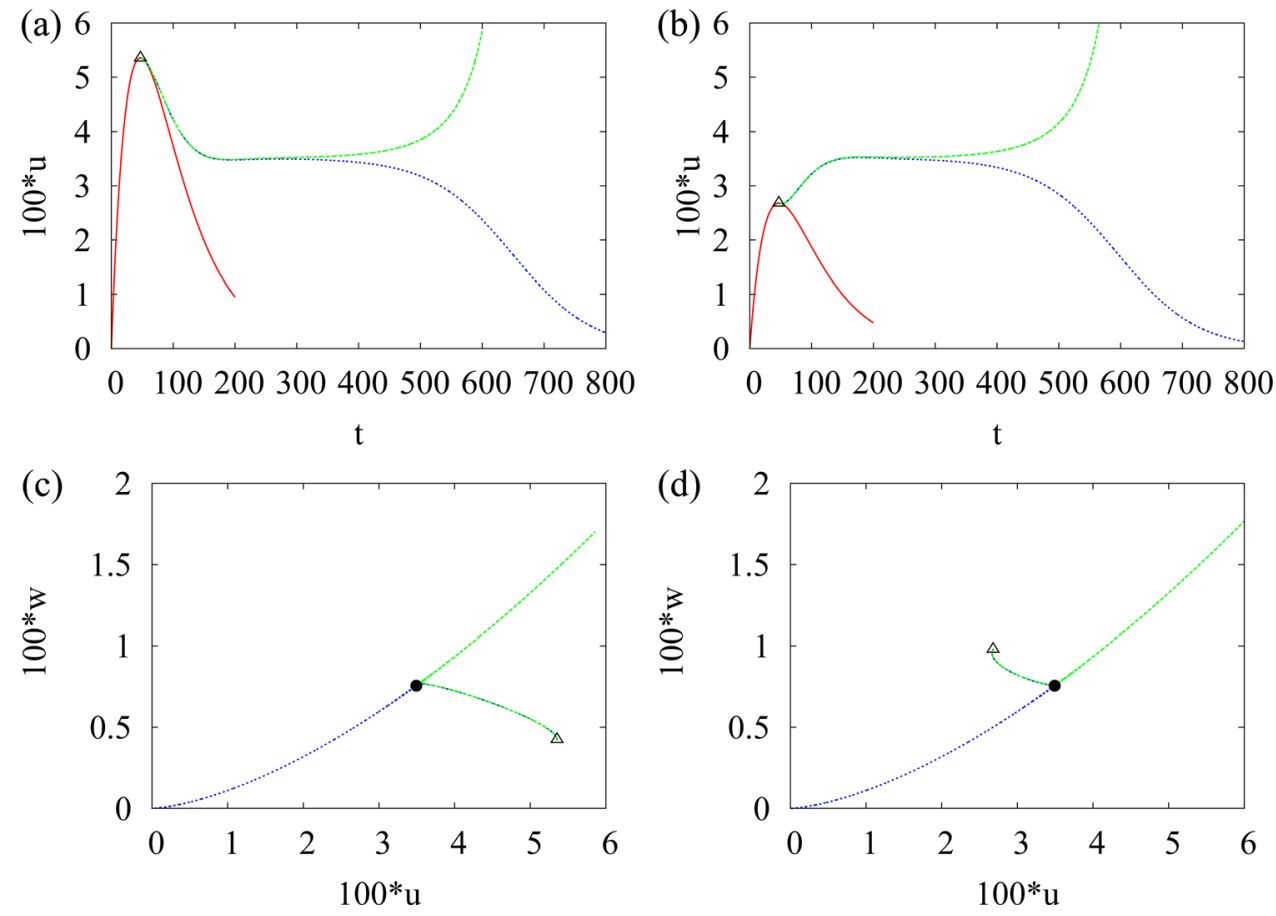

FIG. 1. (Color online) Temporal history of the streaks amplitude $u(t)$ (panels $a$ and $b$ ) and trajectory in the $u-w$ plane (panels $c$ and $d$ ) of selected solutions of Eq. (1) at $R=300$, respectively, corresponding to large (panels $a$ and $c$ ) and small (panels $b$ and $d$ ) initial streak amplitudes. Solid line (red): primary streaks in the absence of secondary perturbations. Dashed line (blue) primary streaks plus marginally stable secondary perturbations. Dotted line (green) primary streaks plus marginally unstable secondary perturbations. The triangles correspond to the time at which the secondary perturbation is added on top of the nonlinearly saturated streaks. The filled circle corresponds to the lower branch saddle solution. secondary perturbation amplitude, while a secondary growth of the streak amplitude $u$ is occurring at the same time (panels $b$ and $d$ in Fig. 1).

The secondary perturbation thresholds have been computed for a full range of primary streak amplitudes and are reported in Fig. 2. This figure conveys the essential message of the paper. Because of the subcritical nature of the sinuous instability of the streaks, the streak breakdown can be achieved for low-amplitude streaks if the amplitude of the secondary perturbations is sufficiently large. To determine the fate of the streamwise streaks, one should therefore consider not only the amplitude of the streaks but also the amplitude of their secondary perturbations, unless the streak amplitudes are definitely supercritical $\left(U \gg U_{c}\right)$. The set of threshold amplitudes (the triangles in Fig. 2) represents a projection on the $u-w$ plane of the boundary of the basin of attraction of the laminar flow in which the lower branch solution $\mathrm{u}_{L B}$ is contained. From the point of view of nonlinear hydrodynamic stability, it is the whole shape of the basin

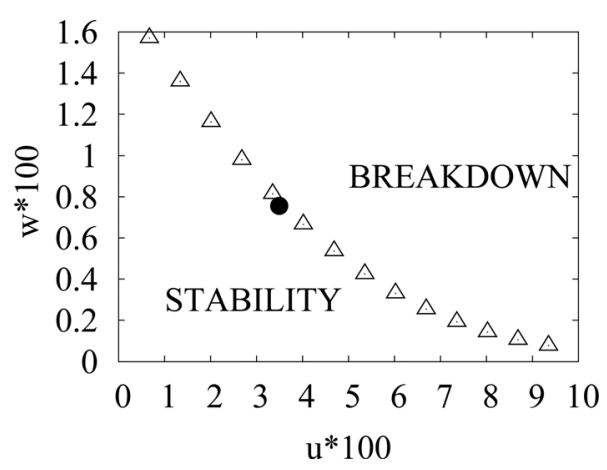

FIG. 2. Secondary sinuous mode threshold amplitudes $w=w_{c}$ necessary to induce breakdown on a primary base flow with streak amplitude $u=U_{1}$ for the model system Eq. (1) at $R=300$. For the considered $R$, the critical amplitude for the (local in time) streak instability is $100 * U_{c}=3.5$. The filled circle symbol corresponds to the lower branch saddle solution found at the same $R$. boundary that is important and not only the lower branch solution. We believe that for real shear flow at large Reynolds number, the shape of the basin boundary is more robust than the exact nature of the edge state (steady, periodic, or chaotic solution).

Interestingly, transition can be obtained even in the almost complete absence of initial streaks and of initial vortices, in accordance with previous findings. ${ }^{31}$ The underlying mechanism can be easily understood in the case of the model system. Consider an initial condition essentially composed of spanwise perturbation $w$ (this corresponds to the far left points in Fig. 2). Upon multiplication by $w$ and defining the new variable $\chi=w^{2}$, Eq. (1c) is transformed into $d \chi / d t \approx-2\left(k_{w}^{2} / R\right) \chi$ (we neglect $u$ and $v$ at small times in this case). Therefore, initially, the energy of the sinuous mode $\chi=w^{2}$ decays, at least as long as the amplitude of the streaks $u$ is small. However, during its decay, the sinuous mode triggers the growth of vortices $v$ through the non-normal coupling in Eq. (1b): $d v / d t=-\left(k_{v}^{2} / R\right) v+\sigma_{v} \chi$. By this mechanism, the vortices quickly attain relatively large amplitudes inducing the growth of the streaks, by standard lift-up, as clearly seen in Fig. 3(a). The phenomenology remains essentially the same in the DNS of transition in Couette and boundary layer flows (see Sec. III). This mechanism, that is essentially active for initial conditions on the left of the saddle point in Fig. 2, cannot be considered as a secondary transient growth on the streaks since $w$ decreases during the process and because there are virtually no primary streaks that would sustain such a secondary growth.

\section{SECONDARY SUBCRITICAL THRESHOLDS IN PLANE COUETTE FLOW}

The concepts discussed on the toy model in Sec. II are now tested on the plane Couette flow of a viscous fluid of kinematic viscosity $\nu$ and constant density $\rho$ between two 


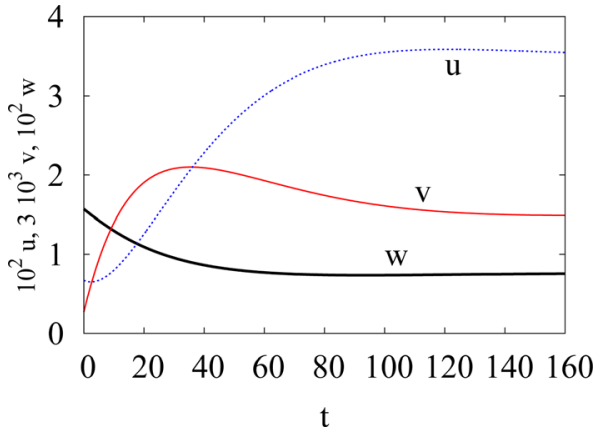

FIG. 3. (Color online) Transient growth of vortices from an initial sinuous perturbations added to weak streaks. Solution obtained for the model system Eq. (1) corresponding to the critical point at lowest $u$ (first point on the left) in Fig. 2. During its initial decay, the sinuous mode (solid thick line, black) induces a transient growth of the vortices (solid line, red) that, in their turn, induce the growth of the streaks (dashed line, blue).

parallel plates located at $y= \pm h$, where we denote by $x, y$, and $z$, the streamwise, wall-normal, and spanwise coordinates, respectively. The plates move in opposite directions with velocity $\left( \pm U_{w}, 0,0\right)$. The flow is assumed periodic in the streamwise and spanwise directions with respective wavelengths $L_{x}$ and $L_{z}$. In the following, we will consider the specific values $L_{x}=2.5 \pi, L_{z}=\pi$, and $R e=500$. These values are similar to the ones used in Ref. $14\left(R e=400, L_{x}=1.75 \pi\right.$, $\left.L_{z}=1.2 \pi\right)$ and Ref. $11\left(\operatorname{Re}=500, L_{x}=2 \pi, L_{z}=\pi\right)$.

The Couette solution $\left(U_{0}(y), 0,0\right)$ with $U_{C}(y)=U_{w} y / h$ is linearly stable for all Reynolds numbers $R e=U_{w} h / \nu$ but sustains large linear transient energy growths $G(t) \equiv$ $\max _{\mathbf{u}_{0} \neq \mathbf{0}}\|\mathbf{u}(t)\|^{2} /\left\|\mathbf{u}_{0}\right\|^{2}$ for the considered parameters. At the linear stage, the different in-plane Fourier modes of streamwise and spanwise wavenumbers $\alpha$ and $\beta$, respectively, can be considered separately. The maximum growth $G_{\text {max }} \equiv \max _{t} G(t)$ is attained, at $t=t_{\text {max }}$, with the optimal initial perturbations $\mathbf{u}_{V}^{(o p t)}$ consisting of streamwise vortices that lead to maximum energy streamwise streaks $\mathbf{u}_{U}^{(o p t)}$ at $t_{\text {max }}$. Standard methods ${ }^{9}$ are used to compute the optimal perturbations for the Couette flow using a Chebyshev-collocation discretization of the Orr-Sommerfeld-Squire equations on a grid of $N_{y}=65$ collocation points in the wallnormal direction.

A family of finite amplitude streaky flows is then built by direct numerical simulation of the nonlinear NavierStokes equations with initial condition $\mathbf{U}_{C}+A_{1} \mathbf{u}_{V}^{(\text {opt })}$, where the linear optimal vortices are given initial finite amplitude $A_{1}$. The DNS are performed using the Simson code ${ }^{32,33}$ it integrates the Navier-Stokes equations, discretized using a Fourier-Chebyshev-Fourier representation in space and a third-order Runge-Kutta method for the nonlinear terms and a second-order Crank-Nicolson method for the linear terms in time. A grid of $32 \times 65 \times 32$ collocation points has been used for the computations discussed below. We consider streamwise uniform $(\alpha=0)$ initial perturbations that induce unsteady solutions $\mathrm{u}_{1}\left(y, z, t, A_{1}\right)$ also streamwise uniform. These solutions consist in streamwise streaks that reach a finite-amplitude maximum energy at times $t$ slightly smaller times than $t_{\text {max }}$. Here, we denote by $\mathrm{U}_{1}\left(y, z, A_{1}\right)$, the streaky velocity field $\mathrm{u}_{1}$ extracted at the time where it reaches its peak perturbation energy.
The velocity fields $\mathrm{U}_{1}\left(y, z, A_{1}\right)$ form a family of nonlinear streamwise uniform streaks whose amplitude is a monotonic function of $A_{1}$. Secondary sinuous perturbations $\mathrm{u}_{W}$ are assumed of the form used by Schoppa and Hussain: ${ }^{17}$

$$
\mathbf{u}_{W}=C\{0,0, g(y) \sin (\alpha x)\} .
$$

with $g(y)=\left(1-y^{2}\right)$ and $C$ a normalizing constant ensuring that $\left\|\mathbf{u}_{W}\right\|=1$.

Also here, when the amplitude of the secondary perturbation is low, the solution relaxes to the laminar Couette solution, while for sufficiently large amplitudes, it leads to the streak breakdown. The critical secondary amplitude is found by a bisection algorithm whose outcome is reported in Fig. 4 for two sample cases with large and small primary streak amplitude. The phenomenology observed is analogous to that described by the toy model in Sec. II. For the largest amplitude of the primary streak (panels $a$ and $c$ of Fig. 4), which is linearly unstable, the threshold amplitude of the secondary perturbation is small, while it is significantly larger for primary streaks that are linearly stable (panels $b$ and $d$ of Fig. 4). For all cases considered, the marginal solutions approach a saddle solution (the Nagata lower branch solution $^{30,34,35}$ ) as shown by the constant rms level of the perturbation. Eventually, the flow transitions to turbulence or relaxes to the laminar Couette solution.

The full set of secondary threshold amplitudes obtained via DNS-based bisection is reported in Fig. 5(a); the behavior is again similar to the picture provided by the toy model.

In the discussion above, we have used the rms values of each velocity component, defined as $u_{r m s}^{2}=\int_{\Omega}\left(u-U_{C}\right)^{2}$ $d \Omega / U_{\text {ref }}^{2}$ and $w_{r m s}^{2}=\int_{\Omega} w^{2} d \Omega / U_{\text {ref }}^{2}$ but the results remain almost unchanged when other measures of the streak and of the secondary perturbation amplitudes are considered. In particular in Fig. 5(b), the critical curve is reported in terms of the amplitudes $A_{U}-A_{W}$ defined as

$$
\begin{gathered}
A_{U}=\left(\max _{y, z} \Delta U-\min _{y, z} \Delta U\right) / 2 U_{r e f} ; \\
A_{W}=\left(\max _{x, y, z} W-\min _{x, y, z} W\right) / 2 U_{r e f},
\end{gathered}
$$

where we choose as reference velocity the difference of the velocity of the two walls: $U_{r e f}=2 U_{w}$. $A_{U}$ is the primary streak amplitude as defined for streamwise uniform streaks ${ }^{13}$ in terms of the maximum and minimum of the distortion of the streamwise velocity $\Delta U(y, z)=U(y, z)-U_{C}(y) . A_{W}$ is defined in a similar way to measure the amplitude of sinuous secondary perturbations. In terms of these amplitudes, the streamwise-uniform streaks become linearly unstable when of $A_{U}=25 \%$. For primary streak amplitudes above this linear critical value, breakdown is obtained with secondary perturbations of amplitude $A_{W} \approx 1 \%$. Such a value is determined as a compromise between the viscous dissipation of the streaks (whose amplitude is not frozen in our simulations), and the time it takes for the instability modes to reach energy levels able to trigger new and more detrimental nonlinear interactions. Only $A_{W} \approx 4 \%$ is necessary to induce breakdown when the primary streak amplitude is decreased to $A_{U} \approx 13 \%$. When further decreasing the amplitude of the 

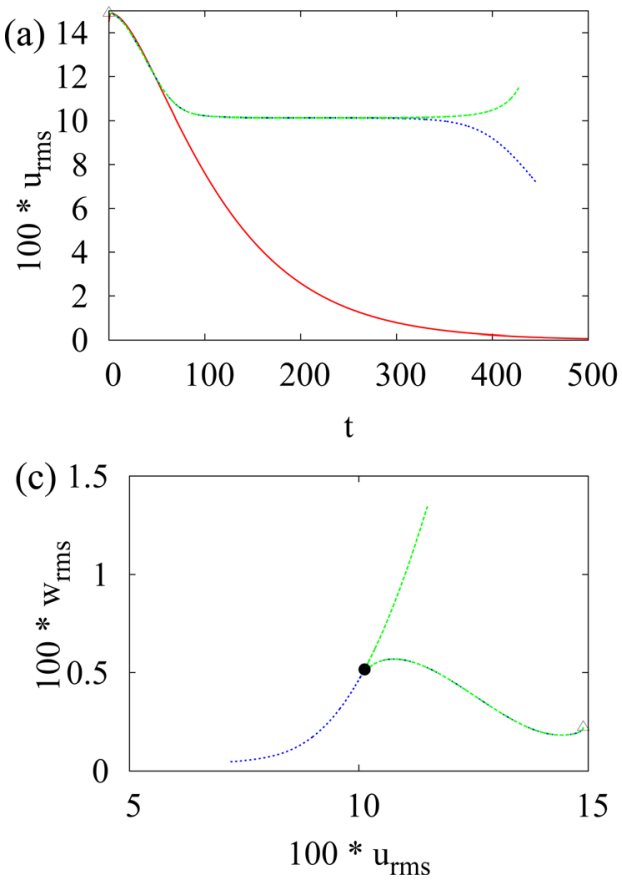
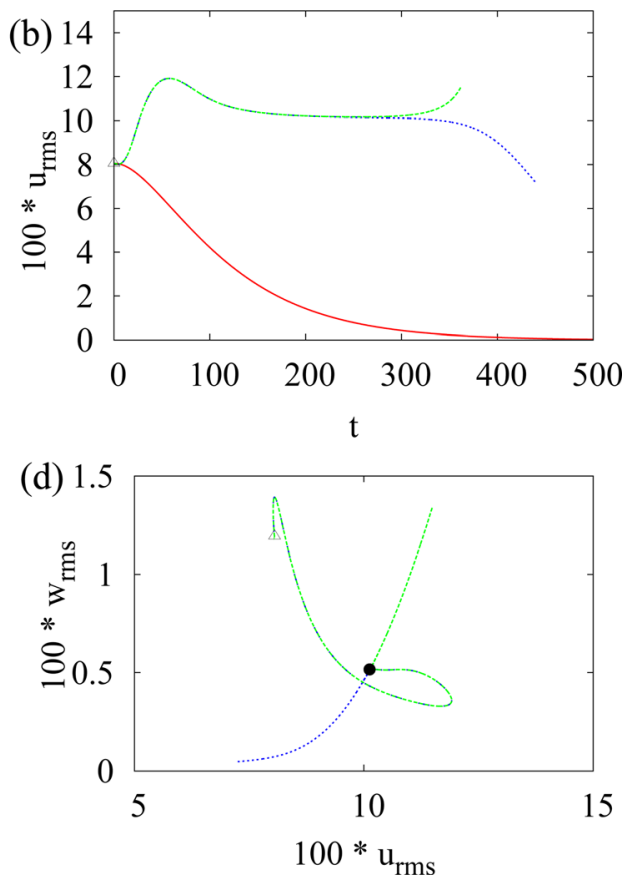

FIG. 4. (Color online) Streak breakdown in Couette flow at $R e=500$. Two cases are considered with, respectively, large (panels $a$ and $c$ ) and small (panels $b$ and $d$ ) initial primary streak amplitude. $(a, b)$ : Temporal history of the rms streamwise velocity perturbation $u_{r m s}$. $(\mathrm{c}, \mathrm{d})$ : Evolution of the solutions in the $u_{r m s}-w_{r m s}$ plane. Solid line (red): primary streaks in the absence of secondary perturbations. Dashed line (blue) primary streaks plus marginally stable secondary perturbations. Dotted line (green) primary streaks plus marginally unstable secondary perturbations. The triangles correspond to the time at which the secondary perturbation is added on top of the nonlinearly saturated streaks. The filled circle corresponds to the lower branch saddle solution. primary streaks below $A_{U} \approx 6 \%$, the amplitude $A_{W}$ of the secondary perturbations needed for the streaks breakdown becomes larger than the amplitude of the primary streaks.

The points on the far left in the plots in Fig. 5 indicate that transition can be induced by an initial secondary sinuous perturbation almost without initial primary streaks or vortices. The transition mechanisms active at very low initial amplitudes of the streaks is not based on a secondary tran-
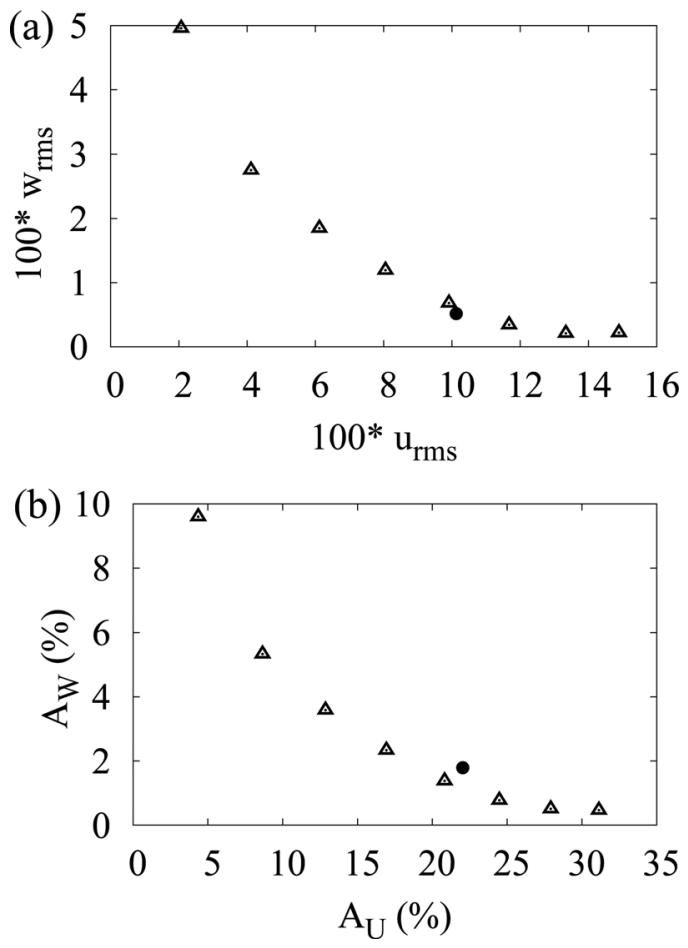

FIG. 5. Threshold amplitudes (triangles) for the sinuous streaks breakdown in Couette flow at $R e=500$ in terms of (a) rms primary streak and secondary perturbations amplitude $u_{r m s}, w_{r m s}$ and (b) of the amplitudes $A_{U^{-}} A_{W}$ defined in Eq. (3). The filled circle symbol corresponds to the lower branch saddle solution at the same Reynolds number. sient growth since the weak primary streaks would not sustain that growth. In this case, a distinct two-stage mechanism is observed, as shown in Fig. 6: the initially decaying sinuous mode (associated with the rms spanwise velocity) forces the growth of streamwise vortices (associated with streamwise vorticity) that then induce the growth and breakdown of streaks (associated with streamwise velocity perturbation and wall-normal vorticity). This mechanism that has been already discussed on the toy model ${ }^{10,24}$ (see Fig. 3) can be easily understood considering the streamwise component of the vorticity transport equation:

$$
\frac{D \omega_{x}}{D t}=\frac{\partial u}{\partial x} \omega_{x}+\frac{\partial u}{\partial y} \omega_{y}+\frac{\partial u}{\partial x} \omega_{z}+\frac{1}{R e} \nabla^{2} \omega_{x},
$$

where $D / D t$ denotes the material derivative. Considering, for simplicity, an initial condition in the complete absence of streaks $u=U_{1}(y), v=0, w=w_{2}(x, y)$ and assuming that the action of viscosity is negligible for short times, then the equation reduces to

$$
\frac{D \omega_{x}}{D t}=\frac{\partial u}{\partial y} \omega_{y}
$$

The tilting of the initial wall-normal vorticity $\omega_{y}=-\partial w_{2} / \partial x$ by the wall normal shear $\partial U_{1} / \partial y$ is responsible for the initial amplification of streamwise vorticity documented in figure 6 . From this figure, it is also seen how the initial pulse of $\omega_{x}$ almost exactly corresponds to the initial pulse of the wall-normal velocity as can be understood either in terms of the standard Biot-Savart law or by taking the wall-normal component of the curl of the vorticity definition $\nabla^{2} v=\partial \omega_{z} / \partial x$ $-\partial \omega_{x} / \partial z$, where the streamwise vortices clearly appear as a source term for the wall-normal velocity. Therefore, the streamwise-periodic vorticity induced by the tilting of the initial wall-normal vorticity disturbance generates on a short time scale streaks of finite length, alternating in a periodic 

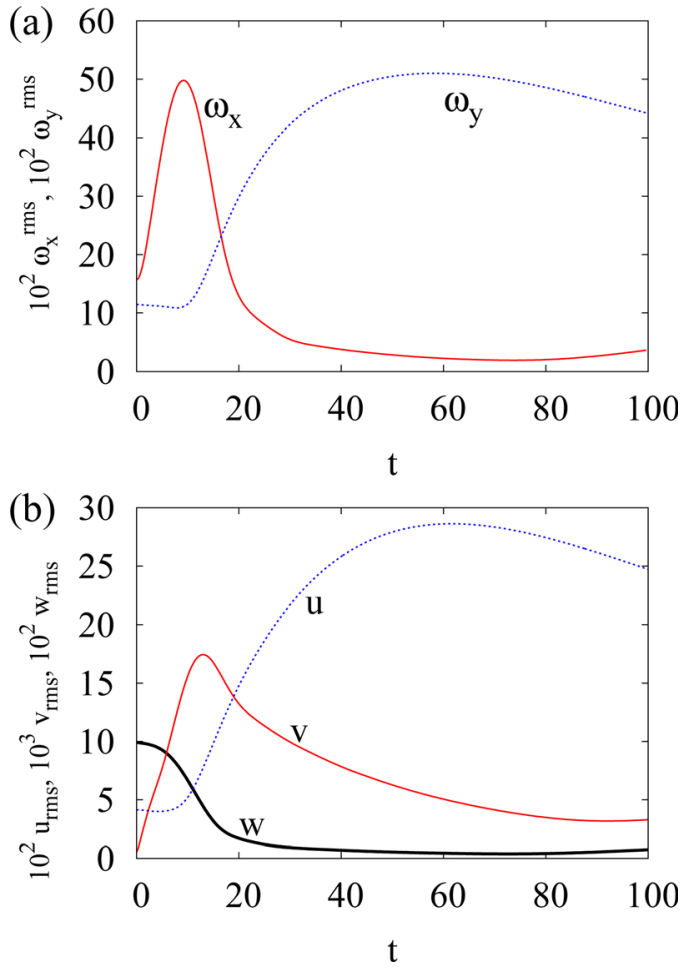

FIG. 6. (Color online) Transient growth of vortices from an initial sinuous perturbations superposed to weak streaks: solution for the Couette flow DNS corresponding to the critical point at lowest $u_{r m s}$ (first point on the left) in Fig. 5. Short-term temporal evolution of the rms streamwise and wall-normal vorticity (panel $a$ ) and streamwise, wall-normal and spanwise velocities $($ panel $b)$.

fashion in the streamwise direction. Indeed, the initial spanwise-velocity perturbation is periodic in the streamwise direction and induces, therefore, streamwise-dependent streamwise vortices. The streaks induced by these vortices also have a finite length and their breakdown to turbulence is associated with the interaction between a downstream low-speed region and an upstream high-speed region that generates strongly inflectional wall-normal profiles. ${ }^{20}$

A final comment is worth about the small offset that can be seen in Fig. 5(b) between the lower branch saddle solution and critical curve. This is due to the fact that the somehow arbitrary initial conditions we use [the saturated straight streak $\mathrm{U}_{1}$ plus the secondary perturbation $A_{2} \mathrm{u}_{W}$ defined in
Eq. (2)] is always different from the lower branch solution. Our critical initial condition, therefore, will be on the basin boundary but always at some finite distance from the lower branch solution. This is why the projection of the lower branch solution is not exactly on the critical curve found using our specific initial conditions.

\section{SECONDARY SUBCRITICAL THRESHOLDS IN BOUNDARY LAYER FLOW}

The second flow we consider in our analysis is the zeropressure-gradient boundary layer with freestream velocity $\left(U_{e}, 0,0\right)$ that is taken as reference velocity $U_{r e f}=U_{e}$. The optimal steady, spanwise periodic perturbations computed in Ref. 36 are used as inflow condition close to the leading edge with amplitude $A_{1}$ and their downstream evolution $\mathrm{u}(x$, $\left.y, z, A_{1}\right)$ is followed by both direct numerical simulations $^{13,26,27}$ and nonlinear parabolized stability equations. ${ }^{37}$ The streaky profiles $U_{1}\left(y, z, A_{1}\right)=U_{0}(y)+u_{1}\left(y, z, A_{1}\right)$ are extracted at the downstream position $x_{\max }$, where the streaks reach maximum energy. The local parallel-flow assumption is used to investigate the stability of the boundary layer profile to initial conditions composed of primary streaks and secondary sinuous perturbations $\mathrm{u}_{W}$ given by Eq. (2) with wall-normal distribution

$$
\left.g(y)=y /\left(\pi \sigma^{2}\right)\right)^{-1 / 2} \exp \left[-\left(\left(y-y_{0}\right)^{2} /\left(\sigma^{2}\right)\right]\right.
$$

with $\sigma=1.7$ and $y_{0}=1.8$. Even if the streaks are allowed to diffuse, a body force is used during the whole simulation in order to prevent the diffusion of the Blasius profile. ${ }^{32,33}$

The fate of the initial conditions is determined by direct numerical simulations assuming the streak profile constant in the streamwise direction and the flow periodic in the streamwise and spanwise directions with respective wavelengths $L_{x}=9 \delta^{*}$ (corresponding to $\alpha \delta^{*}=0.7$ ) and $L_{z}=5.737 \delta^{*}$ (corresponding to $\beta \delta^{*}=1.1$ ) and $R e_{\delta^{*}}=700$. The values of the streamwise wavelength and of the Reynolds number are chosen to reproduce those previously observed in numerical simulations and experiments of transition in boundary layers exposed to free-stream turbulence. ${ }^{38}$ Threshold amplitudes are found by bisection, exactly like in the case of Couette flow. The main difference with the Couette case is that even for very long times, no convergence to a simple edge state
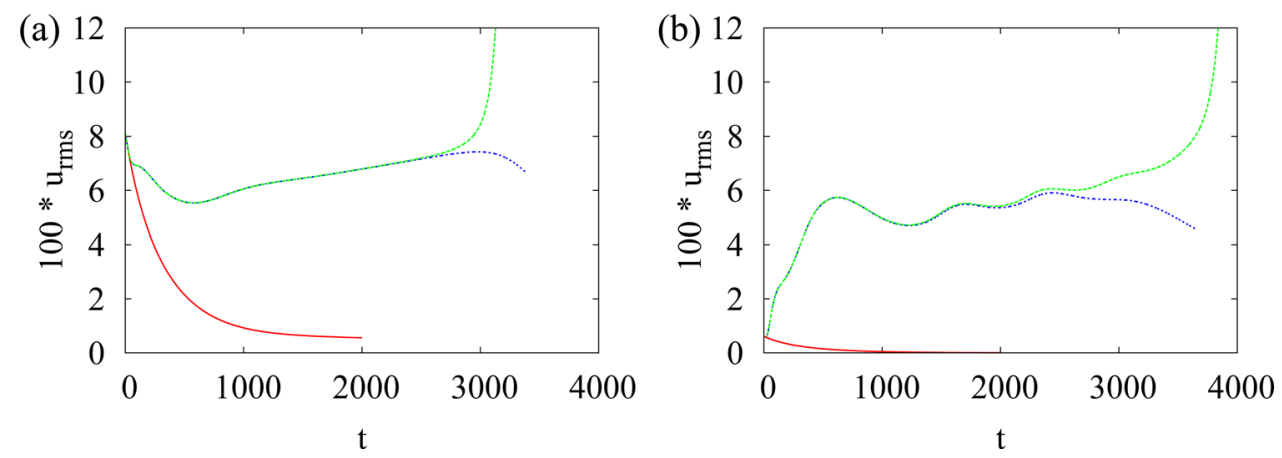

FIG. 7. (Color online) Streak breakdown in Blasius flow at $R e_{\delta^{*}}=700$. Temporal history of the $r m s$ streamwise velocity perturbation $u_{r m s}$ for two cases with, respectively, large (panel $a$, corresponding to the third point from the right in Fig. 9) and small (panel $b$, corresponding to the first point from the left in Fig. 9) initial primary streak amplitude. Solid line (red): primary streaks in the absence of secondary perturbations. Dashed line (blue) primary streaks plus marginally stable secondary perturbations. Dotted line (green) primary streaks plus marginally unstable secondary perturbations. 

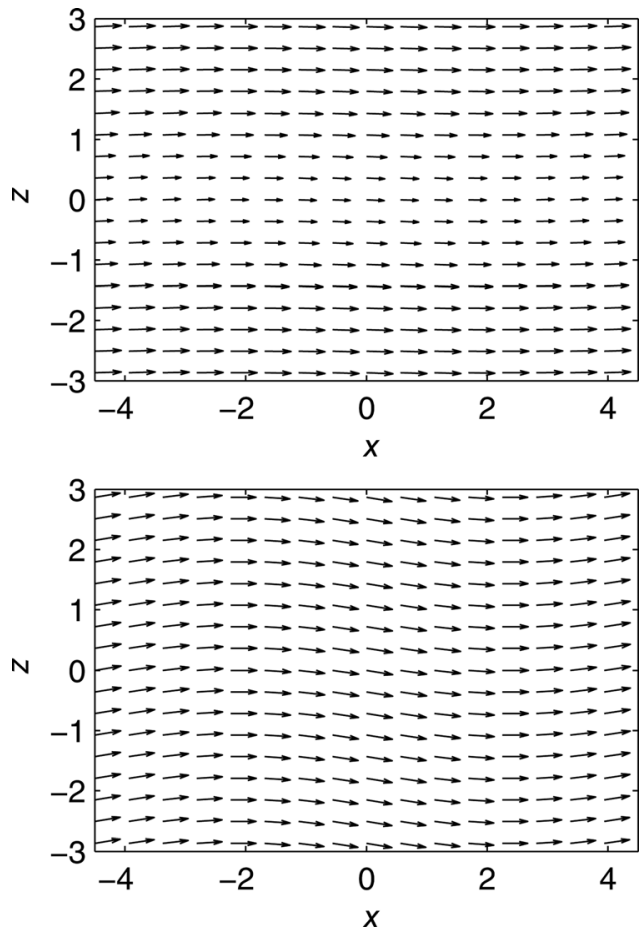

FIG. 8. Velocity field in the wall-parallel plane (u-w components) at $y=2 \delta^{*}$ corresponding to the critical initial conditions in the boundary layer corresponding to: (a) large primary streak amplitude (panel $a$ in Fig. 7) and (b) lowest primary streak amplitude (panel $b$ in Fig. 7). Note that the magnitude of the spanwise velocity component is multiplied by a factor 2 .

such a saddle or limit cycle solution is observed, as can be seen in Fig. 7. No conclusions about the nature of the edge state in the Blasius case can, therefore, be drawn here because extending the computations to even larger times
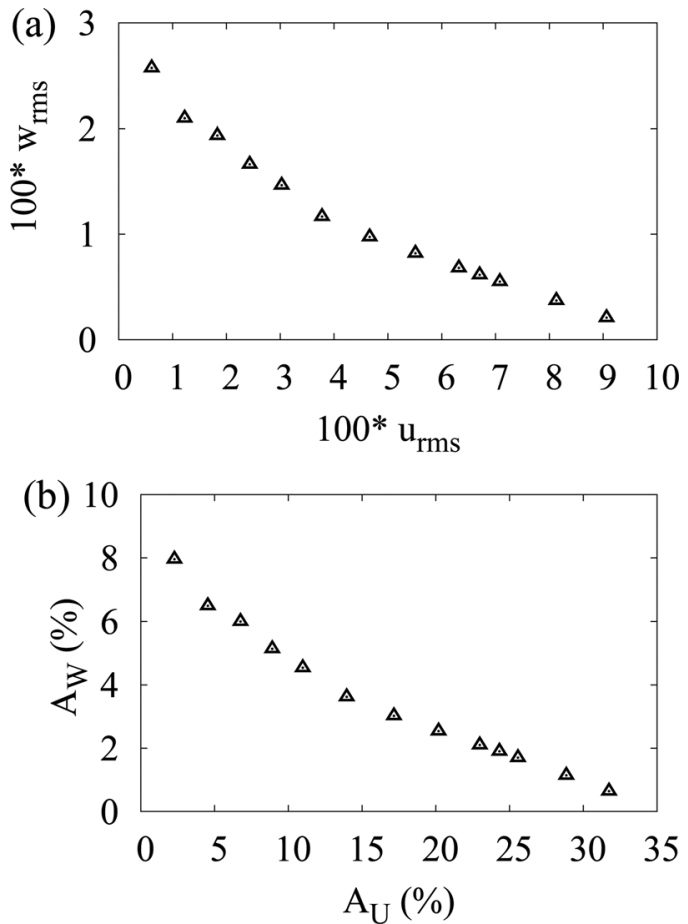

FIG. 9. Threshold amplitudes (triangles) for the sinuous streaks breakdown in the boundary layer flow at $R e_{\delta^{*}}=700$ in terms of (a) rms primary streak and secondary perturbations amplitude $u_{r m s}, w_{r m s}$ and (b) of the amplitudes $A_{U}-A_{W}$ defined in Eq. (3). would clearly violate the parallel flow assumption. This, however, is not really a problem because the critical amplitudes converge on a fast time scale, where the parallel flow assumption holds; in addition, here we focus on the shape of the basin boundary more than on the nature of the edge states lying in it. The determination of the edge state in the fully non-parallel case has been recently considered elsewhere. ${ }^{39}$

The marginal initial conditions pertaining to two cases with, respectively, low and large initial streak amplitude are shown in Fig. 8. The velocity vector field is displayed in the wall-parallel plane at $y / \delta^{*}=2$. Note that the spanwise velocity is amplified by a factor 2 : indeed relatively low bending is necessary to trigger the laminar-turbulent transition.

The results of the bisection procedure, reported in Fig. 9 , reproduce the trends and considerations of the Couette flow, as well as those of the toy model. The critical $A_{U}-A_{W}$ boundary is also quantitatively similar to the one found for the Couette case, despite the important differences between the two flows.

\section{SUMMARY AND CONCLUSIONS}

In this study, we have explicitly computed by direct numerical simulation the nonlinear stability of the Couette and boundary-layer laminar solutions with respect to initial conditions consisting of a primary streamwise-uniform streak and secondary sinuous spanwise perturbation.

The critical curve, found by bisection using direct numerical simulations, has been computed in terms of the amplitude of the streamwise streak and of the amplitude of the sinuous perturbation. This critical curve is a two-dimensional projection of the shape of the boundary of the basin of attraction of the laminar state for the considered subcritical bifurcations.

The different streak transition scenarios that have been considered in previous investigations correspond to different parts of the threshold curve. Transition due to modal instability ${ }^{10-13}$ of the streaks is found for large streak amplitudes $\left(A_{U}=25 \%-27 \%\right)$, where small amplitudes of the secondary perturbation $\left(A_{W}<1 \%-2 \%\right)$ are sufficient to overcome the streak decay and trigger the breakdown. Transition based on streaks transient growth ${ }^{17-19}$ is found for smaller values of $A_{U}$, where larger amplitudes of secondary perturbations are required for transition. For the plane Couette flow case, the solutions approach the lower branch saddle solution ${ }^{30,34,35}$ in the transition process, which represents an edge state. These lower branch solutions, however, represent a single point on the boundary of the basin of attraction of the laminar solution. From the nonlinear stability perspective, the whole critical curve is of interest to assess the stability of the laminar flow.

For streamwise streaks amplitudes below $A_{U} \approx 6 \%$, spanwise perturbations of amplitude larger than that of the streak are required for breakdown. In this case, secondary transient growth is inactive because the weak primary streaks would not sustain that growth. A new two-stage mechanism has been found to be operating in this range: the initially decaying sinuous mode forces the growth of vortices that then induce the growth of streaks. This interpretation has 
been devised on Waleffe's reduced model ${ }^{10,24}$ and confirmed by the analysis of the DNS for both Couette and boundarylayer flow.

The computation of the transition threshold curve for the streak breakdown in terms of the amplitude of the streaks and of the sinuous perturbations is probably also relevant for the design of control protocols aimed at, e.g., suppressing transition by reducing the amplitude of the streaks or at delaying transition using streaks. ${ }^{26,40}$ Due to the artificial type of perturbations we have considered (spatially periodic with a specific initial shape), some caution is recommended in the direct application of the computed critical curves to real world experiments. Also, localized perturbations, typical of natural transition, have not been considered here. However, with all these words of caution, we believe that the main conclusions of the present study still apply, at least qualitatively:for the control to be robust, the level of spanwise perturbations should also be kept particularly low.

An important question is to know in which part of the $A_{U}$ $-A_{W}$ critical curve, natural transition, like transition induced by free-stream turbulence, is likely to be observed. If the external perturbations, or the nonlinear terms in the NavierStokes equations, can be modeled as a stochastic forcing term or as a noisy initial condition, then it is known that the amplification of streamwise uniform perturbations (the streaks) is larger than the amplification of spanwise perturbations with finite streamwise wavelength and that the difference in amplification increases with the Reynolds number. When the Reynolds number is large, therefore, one would expect to observe the "classical" modal scenario, where the streaks are largely amplified and become locally modally unstable. Sustained amplification of low-amplitude transverse perturbations would then initiate the breakdown. However, bypass transition is usually observed at Reynolds numbers that are not asymptotically large, where the amplification of the streaks is not likely to be more than one order of magnitude larger than the amplification of transverse perturbations. In such a situation, it is perfectly likely, and it is indeed observed, that transition can occur even when the streamwise streaks are locally stable. To fully understand the transition scenario, experimental and numerical data could be processed not only with the aim of computing the streamwise streaks amplitude but also the amplitude of secondary perturbations, and the results checked versus the critical curve we have computed. This also applies to the interpretation of the self-sustained processes in turbulent shear flows. In that case, is it also likely that the effective Reynolds number, based on some sort of eddy viscosity, is relatively low and therefore that low amplitude unsteady streaks are observed. This is indeed the case that has motivated previous investigations. ${ }^{17}$ One could, therefore, conjecture that bursting events correspond to forcing of the streaks by other structures, with spanwise perturbations above the critical threshold.

In the present investigation, we have considered only sinuous perturbations because they are the ones that first become unstable when the primary streak amplitude is increased. However, for streaks different from the one considered here, which are issued from optimal initial vortices, it could be that the first unstable modes are varicose. In that case, of course the results of the present study do not apply, and appropriate critical curves should be recomputed using the same rationale.

\section{ACKNOWLEDGMENTS}

C. C. and D. H. acknowledge the kind hospitality of the Linné FLOW Center and of IMFT where parts of this study have been conducted.

${ }^{1}$ G. I. Taylor, in Proceedings of the 5th International Congress Application Mechanism, edited by J. Hartog and H. Peters (Wiley, New York, 1939) pp.294-310.

${ }^{2} \mathrm{P}$. S. Klebanoff, "Effect of free-stream turbulence on the laminar boundary layer," Bull. Am. Phys. Soc. 10, 1323 (1971).

${ }^{3} \mathrm{D}$. Arnal and J. C. Juillen, "Contribution expérimentale à l'étude de la receptivité d'une couche limite laminaire à la turbulence de l'écoulement général," Rapport Technique 1/5018 ONERA, 1978,

${ }^{4}$ S. J. Kline, W. C. Reynolds, F. A. Schraub, and P. W. Runstadler, "The structure of turbulent boundary layers," J. Fluid Mech. 30, 741-773 (1967).

${ }^{5} \mathrm{H}$. K. Moffatt, in Proceedings of the URSI-IUGG Colloquium on Atoms, Turbulence and Radio Wave Propagation, edited by A. Yaglom and V. I. Tatarsky (Nauka, Moscow, 1967) pp.139-154.

${ }^{6} \mathrm{M}$. T. Landahl, "A note on an algebraic instability of inviscid parallel shear flows," J. Fluid Mech. 98, 243 (1980).

${ }^{7}$ L. H. Gustavsson, "Energy growth of three-dimensional disturbances in plane Poiseuille flow," J. Fluid Mech. 224, 241 (1991).

${ }^{8}$ L. N. Trefethen, A. E. Trefethen, S. C. Reddy, and T. A. Driscoll, "A new direction in hydrodynamic stability: Beyond eigenvalues," Science 261, 578 (1993).

${ }^{9}$ P. J. Schmid and D. S. Henningson, Stability and Transition in Shear Flows (Springer, New York), 2001.

${ }^{10} \mathrm{~F}$. Waleffe, "Hydrodynamic stability and turbulence: Beyond transients to a self-sustaining process," Stud. Appl. Math. 95, 319 (1995).

${ }^{11}$ S. C. Reddy, P. J. Schmid, J. S. Baggett, and D. S. Henningson, "On the stability of streamwise streaks and transition thresholds in plane channel flows," J. Fluid Mech. 365, 269 (1998).

${ }^{12}$ G. Kawahara, J. Jiménez, M. Uhlmann, and A. Pinelli, "The instability of streaks in near-wall turbulence," Tech. Report (NASA-Stanford University Center for Turbulence Research, Annual Research Briefs, 1998) pp.155170 .

${ }^{13}$ P. Andersson, L. Brandt, A. Bottaro, and D. Henningson, "On the breakdown of boundary layers streaks," J. Fluid Mech. 428, 29 (2001).

${ }^{14}$ J. Hamilton, J. Kim, and F. Waleffe, "Regeneration mechanisms of nearwall turbulence structures," J. Fluid Mech 287, 317 (1995).

${ }^{15} \mathrm{~F}$. Waleffe, "Transition in shear flows. nonlinear normality versus non-normal linearity," Phys. Fluids 7, 3060 (1995).

${ }^{16}$ G. Kreiss, A. Lundbladh, and D. S. Henningson, "Bounds for threshold amplitudes in subcritical shear flows," J. Fluid Mech. 270, 175 (1994).

${ }^{17} \mathrm{~W}$. Schoppa and F. Hussain, "Coherent structure generation in near-wall turbulence," J. Fluid Mech. 453, 57 (2002).

${ }^{18}$ J. Hopffner, L.Brandt, and D. S.Henningson, "Transient growth on boundary layer streaks," J. Fluid Mech. 537, 91 (2005).

${ }^{19} \mathrm{C}$. Cossu, M. Chevalier, and D. Henningson, "Optimal secondary energy growth in a plane channel flow," Phys. Fluids 19, 058107 (2007).

${ }^{20} \mathrm{~L}$. Brandt and H. C. de Lange, "Streak interactions and breakdown in boundary layer flows," Phys. Fluids 20, 024107 (2008).

${ }^{21}$ N. J. Vaughan and T. Zaki, "Stability of zero-pressure-gradient boundary layer distorted by unsteady klebanoff streaks," J. Fluid Mech. (in press).

${ }^{22} \mathrm{~F}$. Waleffe, "Three-dimensional coherent states in plane shear flows," Phys. Rev. Lett. 81, 4140 (1998).

${ }^{23} \mathrm{~F}$. Waleffe, "Exact coherent structures in channel flow," J. Fluid Mech. 435, 93 (2001).

${ }^{24} \mathrm{~F}$. Waleffe, "On a self-sustaining process in shear flows," Phys. Fluids $\mathbf{9}$, 883 (1997).

${ }^{25}$ D. Henningson, "Comment on "transition in shear flows. nonlinear normality versus non-normal linearity," [Phys. Fluids 7, 3060 (1995)]," Phys. Fluids 8, 2257 (1996).

${ }^{26}$ C. Cossu and L. Brandt, "Stabilization of Tollmien-Schlichting waves by finite amplitude optimal streaks in the Blasius boundary layer," Phys. Fluids 14, L57 (2002). 
${ }^{27}$ L. Brandt, C. Cossu, J.-M. Chomaz, P. Huerre, and D. S. Henningson, "On the convectively unstable nature of optimal streaks in boundary layers," J. Fluid Mech. 485, 221 (2003).

${ }^{28}$ B. Eckhardt, T. Schneider, B. Hof, and J. Westerweel, "Turbulence Transition in Pipe Flow," Ann. Rev. Fluid Mech. 39, 447 (2007).

${ }^{29}$ T. M. Schneider, B. Eckhardt, and J. A. Yorke, "Turbulence transition and the edge of chaos in pipe flow," Phys. Rev. Lett. 99, 034502 (2007).

${ }^{30}$ T. Schneider, J. Gibson, M. Lagha, F. De Lillo, and B. Eckhardt, "Laminar-turbulent boundary in plane Couette flow," Phys. Rev. E 78, 37301 (2008).

${ }^{31}$ C. Cossu, M. Chevalier, and D. Henningson, in Seventh IUTAM Symposium on Laminar-Turbulent Transition, edited by P. Schlatter and D. Henningson (Springer, Berlin, 2010), pp.129-134.

${ }^{32}$ A. Lundbladh, D. S. Henningson, and A. V. Johansson, "An efficient spectral integration method for the solution of the Navier-Stokes equations," Tech. Rep. FFA, the Aeronautical Research Institute of Sweden, 1992.

${ }^{33}$ M. Chevalier, P. Schlatter, A. Lundbladh, and D. S. Henningson, "A Pseudo-Spectral Solver for Incompressible Boundary Layer Flows,” Tech.
Report No. TRITA-MEK 2007:07 Royal Institute of Technology KTH, Dept. of Mechanics, Stockholm, 2007.

${ }^{34}$ M. Nagata, "Three-dimensional finite-amplitude solutions in plane Couette flow: bifurcation from infinity," J. Fluid Mech. 217, 519 (1990).

${ }^{35} \mathrm{~F}$. Waleffe, "Homotopy of exact coherent structures in plane shear flows," Phys. Fluids 15, 1517 (2003).

${ }^{36} \mathrm{P}$. Andersson, M. Berggren, and D. Henningson, "Optimal disturbances and bypass transition in boundary layers," Phys. Fluids 11, 134 (1999).

${ }^{37}$ S. Bagheri and A. Hanifi, "The stabilizing effect of streaks on TollmienSchlichting and oblique waves: A parametric study," Physics of Fluids 19, 078103 (2007).

${ }^{38}$ P.Schlatter, L. Brandt, H. C. de Lange, and D. S. Henningson, "On streak breakdown in bypass transition," Phys. Fluids 20 (2008).

${ }^{39}$ S. Cherubini, P. D. Palma, J.-C. Robinet, and A. Bottaro, "Edge states in a boundary layer," Phys. Fluids 23, 051705 (2011).

${ }^{40}$ J. Fransson, L. Brandt, A. Talamelli, and C. Cossu, "Experimental study of the stabilisation of Tollmien-Schlichting waves by finite amplitude streaks," Phys. Fluids 17, 054110 (2005). 